\title{
SCUBA sub-millimeter observations of gamma-ray bursts
}

\section{GRB 021004, 021211, 030115, 030226, 041006}

\author{
I. A. Smith ${ }^{1}$, R. P. J. Tilanus ${ }^{2}$, N. Tanvir ${ }^{3}$, V. E. Barnard ${ }^{2}$, G. H. Moriarty-Schieven ${ }^{2}$, D. A. Frail ${ }^{4}$, \\ R. A. M. J. Wijers ${ }^{5}$, P. Vreeswijk ${ }^{6}$, E. Rol ${ }^{7}$, and C. Kouveliotou ${ }^{8,9}$ \\ 1 Department of Physics and Astronomy, Rice University, 6100 South Main, MS-108, Houston, TX 77005-1892, USA \\ e-mail: iansmith@rice.edu \\ 2 Joint Astronomy Centre, 660 N. Aohoku Place, Hilo, HI 96720, USA \\ 3 Centre for Astrophysics Research, University of Hertfordshire, College Lane, Hatfield, Herts AL10 9AB, UK \\ ${ }^{4}$ National Radio Astronomy Observatory, PO Box O, Socorro, NM 87801, USA \\ 5 Astronomical Institute "Anton Pannekoek", University of Amsterdam and Center for High-Energy Astrophysics, \\ Kruislaan 403, 1098 SJ Amsterdam, The Netherlands \\ ${ }^{6}$ European Southern Observatory, Alonso de Córdova 3107, Casilla 19001, Santiago 19, Chile \\ 7 Department of Physics and Astronomy, University of Leicester, Leicester LE1 7RH, UK \\ 8 NASA Marshall Space Flight Center, SD-50, NSSTC, 320 Sparkman Drive, Huntsville, AL 35805, USA \\ ${ }^{9}$ Universities Space Research Association
}

Received 22 March 2005 / Accepted 10 May 2005

\begin{abstract}
We discuss our ongoing program of Target of Opportunity (ToO) sub-millimeter observations of gamma-ray bursts (GRBs) using the Sub-millimetre Common-User Bolometer Array (SCUBA) on the James Clerk Maxwell Telescope (JCMT). In this paper, we present the ToO observations of GRBs 021004, 021211, 030115, 030226, and 041006. The observations of GRBs 021004, 021211, 030226, and 041006 all started within $\sim 1$ day of the burst, but did not detect any significant sub-millimeter emission from the reverse shock and/or afterglow. These observations put some constraints on the models for the early emission, although the generally poor observing conditions and/or the faintness of these afterglows at other wavelengths limit the inferences that can be drawn from these lack of detections. However, these observations demonstrate that SCUBA can perform rapid observations of GRBs, and provide encouragement for future observations in the Swift era. None of these GRBs had significant sub-millimeter emission from their host galaxies. This adds to the indication that GRBs are not closely linked to the most luminous dusty star-forming galaxies.
\end{abstract}

Key words. gamma rays: bursts - submillimeter

\section{Introduction}

The discovery of localized transients in the error boxes of gamma-ray burst (GRB) sources has led to intense multiwavelength campaigns that have revolutionized our understanding of these sources. For reviews see Van Paradijs et al. (2000) and Mészáros (2002). The current evidence indicates that at least some GRBs are due to the explosive collapse of massive stars.

During and after the burst, the observed multiwavelength emission comes from several distinct components. The explosion produces shocks that energize particles whose radiation gives the "prompt" bright burst emission. A reverse shock can give an optical and/or radio "flash". The "afterglow" emission comes from an expanding fireball as it sweeps up the surrounding medium. At later times, signatures may appear that are characteristic of supernovae or hypernovae. Finally, the "quiescent" constant emission comes from any underlying host galaxy.

Sub-millimeter observations form a key element of the multiwavelength observations of the bursts. They provide "clean" measures of the source intensity, unaffected by scintillation and extinction. To this end, we have been performing Target of Opportunity (ToO) sub-millimeter observations of GRB counterparts using the Sub-millimetre Common-User Bolometer Array (SCUBA) on the James Clerk Maxwell Telescope (JCMT) on Mauna Kea, Hawaii.

The detailed SCUBA ToO results for the first eight bursts studied (GRBs 970508, 971214, 980326, 980329, 980519, 980703, 981220, and 981226) are described in Smith et al. (1999); GRB 980329 is also discussed in Yost et al. (2002) and Berger et al. (2003), and GRB 980703 in Bloom et al. (1998) and Frail et al. (2003a). GRB 990123 is discussed in Galama et al. (1999) and Kulkarni et al. (1999). Observations 
of GRB 990520 were made in mediocre weather (Smith et al. 2000). The next six bursts (GRBs 991208, 991216, 000301C, 000630, 000911, and 000926) are described in Smith et al. (2001); GRB 991216 is also discussed in Frail et al. (2000), and GRB 000301C in Berger et al. (2000). GRB 010222 was described in Frail et al. (2002). In this paper, we present all the ToO observations of GRBs made by SCUBA from March 2001 through April 2005, with the exception of GRB 030329, which was discussed in Smith et al. (2005). The results shown here are for GRBs 021004, 021211, 030115, 030226, and 041006.

In the remainder of Sect. 1, we outline the motivations for making sub-millimeter observations of the afterglows and host galaxies and summarize some of the previous results. In Sect. 2 we describe the most relevant technical details of the SCUBA observations and data analysis. In Sect. 3 we present the results for our latest SCUBA ToO observations. In Sect. 4 we discuss the results. In Sect. 5 we explain how our program will make good use of the bursts observed by Swift.

\subsection{SCUBA afterglow observations}

Both observations (e.g. Galama et al. 1998, 1999; Smith et al. 2005) and theories (e.g. Sari et al. 1998; Piran 1999; Wijers \& Galama 1999; Granot et al. 2000; Sari \& Mészáros 2000; Chevalier \& Li 2000; Granot \& Sari 2002; Panaitescu \& Kumar 2004; Inoue et al. 2005) show that for some bursts the reverse shock and/or afterglow emission can peak in the sub-millimeter in the hours to weeks following the burst.

By tracking the evolving emission across the entire spectrum, it is possible to study aspects such as the types of shocks involved, the geometry of the outflow (jet versus spherical), and the geometry of the surrounding medium (uniform versus stellar wind). It is of interest to look for variations in the afterglow light curve that could be due to the refreshing of the shock in the fireball (e.g. Sari \& Mészáros 2000; Granot et al. 2003), or due to inhomogeneities in the ambient medium that the fireball is expanding into (e.g. Berger et al. 2000).

The afterglow evolution can be complex, and separating the different components is not trivial. Sub-millimeter observations performed within a day of the burst are of particular importance, since they can potentially strongly discriminate between different afterglow models (Panaitescu \& Kumar 2000; Livio \& Waxman 2000; Yost et al. 2003; Inoue et al. 2005). Distinct early evolution behaviors could also be present in the radio data, but they might be hard to extract due to scintillation and/or self-absorption. At X-ray/optical frequencies, the difference in the early evolution is much smaller, and suffers from degeneracies.

The sub-millimeter flux from the forward shock in a fireball that is interacting with a homogeneous medium should evolve relatively slowly over the first few days after the burst. However, for a fireball that is interacting with a prior stellar wind, the forward shock is propagating down a large density gradient and the sub-millimeter flux will rapidly rise and fall. After a few days, the evolution of the sub-millimeter flux will be similar for both models. The contrasting behavior of these two scenarios is illustrated in Fig. 1.

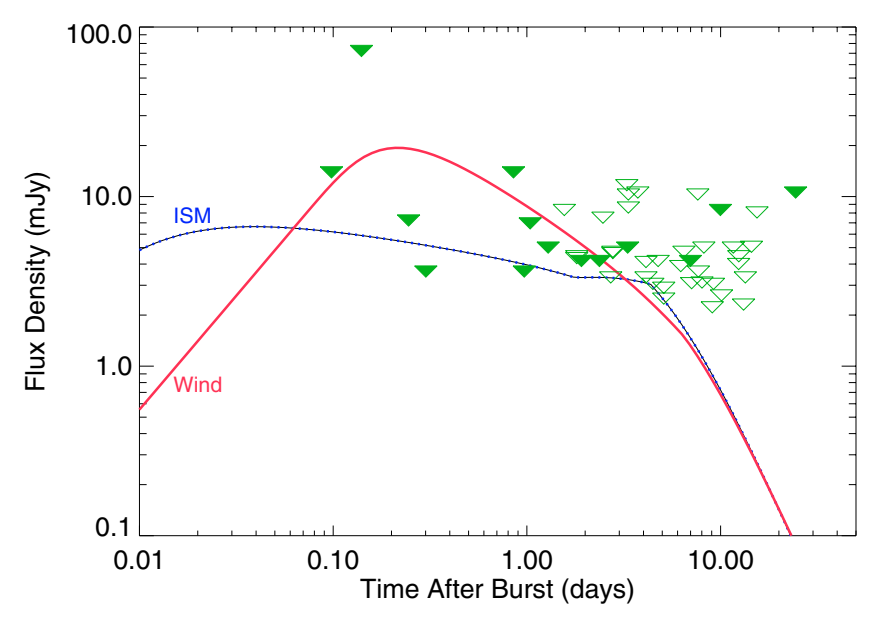

Fig. 1. The model curves illustrate the difference in the $850 \mu \mathrm{m}$ evolution expected for a GRB that explodes in a wind-fed (Wind) or a constant density (ISM) circumburst medium. The models are taken from fits made to the $\mathrm{cm}$ radio, near-IR, optical, and X-ray data of GRB 980703 and redshifted to $z=1.5$, which is typical for the GRBs observed to date (Frail et al. 2003a). The models only use a forward shock; an additional component from a reverse shock may also be present at early times. The $3 \sigma$ upper limits show all the $850 \mu \mathrm{m}$ GRB ToO observations made by SCUBA where no afterglow was detected. The filled triangles show the data for the new bursts presented in this paper. These new observations are starting to meaningfully sample the sub-millimeter emission at early times where there can be significant differences in the evolution of the sub-millimeter flux in the wind and ISM scenarios.

There may also be a significant sub-millimeter emission at early times from a reverse shock. The wavelength for the peak of the reverse shock emission and the flux at the peak may be relatively insensitive to the redshift of the burst (Inoue et al. 2005). Thus the reverse shock emission may be at mJy levels out to $z \sim 30$, even if the forward shock is too faint to detect.

The most significant afterglow emission observed by SCUBA to date was from GRB 030329 (Smith et al. 2005). This had a $\sim 31 \mathrm{mJy}$ flux density at $850 \mu \mathrm{m}$ that was essentially constant up to $\sim 7$ days after the burst. After this it had a break to a steep decay. The $850 \mu \mathrm{m}$ results agreed with those at longer wavelengths that showed a brighter flux $\sim 7$ days after the burst, right at the time of the break. No short-lived large-scale brightenings were detected in the sub-millimeter light curve. However, the $850 \mu \mathrm{m}$ light curve may have had a drop $\lesssim 16$ days after the burst. The peak of the afterglow emission was at $\sim 90 \mathrm{GHz}$ in the days before the break in the light curve. A simple modeling was consistent with the spectral indices remaining the same as the afterglow evolved, with the breaks in the spectrum moving to longer wavelengths at later times and the flux at the peak falling.

Prior to GRB 030329, the most significant afterglow emission observed by SCUBA at $850 \mu \mathrm{m}$ was from GRB 980329 (Smith et al. 1999; Yost et al. 2002; Berger et al. 2003). The flux and rms reported by Smith et al. were higher than in the later papers. This is because Smith et al. dropped the last two (out of five) runs from the analysis on 1998 April 5 and the third one (out of five) from 1998 April 6. In both cases, the declining flux during these runs suggested a pointing drift; on April 6, 
a re-pointing and re-focusing after the third run resulted in the signal returning in the remaining runs. Searches for a host galaxy indicate that its contribution is $<1 \mathrm{mJy}$ (Tanvir et al. 2004). In conclusion, the fading afterglow for GRB $980329 \mathrm{had}$ an $850 \mu$ m flux density $\sim 3 \mathrm{mJy}$ one week after the burst.

Some of the ToO observations of other bursts have produced possible detections at $850 \mu \mathrm{m}$. For GRB 990123, there was a $3.3 \sigma$ detection of 4.9 mJy on 1999 February 4, although this was not detected in longer observations the following night or in earlier observations (Galama et al. 1999; Kulkarni et al. 1999). For GRB 000301C, there was an indication of a $\sim 3 \mathrm{mJy}$ source at $850 \mu \mathrm{m}$, but only on 2000 March 5 (Smith et al. 2001). This was at the time when an achromatic brightening was found at other wavelengths (e.g. Masetti et al. 2000; Berger et al. 2000). The limited observation of GRB 000926 gave $7.3 \pm 4.2 \mathrm{mJy}$ at $850 \mu \mathrm{m}$ on 2000 September 30 . This is consistent with the multiwavelength observations of Harrison et al. (2001) that showed the peak of the spectrum was in the sub-millimeter at that time. The sub-millimeter emission from GRB 010222 may have had a $\sim 1$ mJy contribution from the afterglow (Berger et al. 2003).

Prior to this paper, GRB 010222 was the only case where the initial SCUBA observation was performed within one day of the burst. There has therefore been limited study of the potentially bright early sub-millimeter emission from the afterglow and/or the reverse shock.

\subsection{SCUBA host galaxy observations}

In addition to studying the afterglows, it is important to understand the nature of the host galaxies containing the GRBs.

It is plausible that if GRBs are due to the explosive deaths of high-mass stars, they are likely to be found in active star forming regions. Optical studies indicate that many of the host galaxies studied to date are similar to young starburst galaxies with moderate to low extinction (e.g. Fynbo et al. 2003; Christensen et al. 2004). Sub-millimeter observations can investigate whether there is a connection between GRBs and dust-enshrouded star formation (Blain \& Natarajan 2000). If so, estimates have suggested that $\sim 20 \%$ of GRB hosts will be brighter than $2 \mathrm{mJy}$ at $850 \mu \mathrm{m}$ (Ramirez-Ruiz et al. 2002) or that $7-18 \%$ will be brighter than $4 \mathrm{mJy}$ at $850 \mu \mathrm{m}$ (Trentham et al. 2002).

SCUBA has discovered (non-GRB) sub-millimeter bright galaxies out to high redshifts; for a review see Blain et al. (2002). It appears that the star formation rate does not drop rapidly beyond $z \sim 1$ (e.g. Blain et al. 1999), and the median redshift for galaxies that are bright at sub-millimeter wavelengths is $\sim 2.3$ (Chapman et al. 2003, 2005). Many of the submillimeter bright sources may be undergoing major mergers (Conselice et al. 2003).

The relatively small population of extremely luminous dusty galaxies may dominate the total star formation in the universe at early epochs. However, a difficulty in reaching this conclusion is that there may be a substantial contribution to the energy output of the SCUBA-bright galaxies from active galactic nuclei. Furthermore, some galaxies are sub-millimeter bright simply due to gravitational lensing (e.g. Dunlop et al. 2004). If GRBs are regularly found to be associated with extremely luminous sub-millimeter bright dusty galaxies, this could provide independent evidence that these objects dominate the star formation in the early universe. The gamma-rays from the bursts can be detected out to high redshift, and through large columns of dust and gas.

Our ToO program is designed to look for the afterglow emission of the bursts by making observations shortly after the burst, with follow-up observations on subsequent days. Since these ToO observations are done on short notice, their sensitivity can be limited by non-optimal weather and/or source location in the sky. However, by combining the data from all of our ToO observations of a source we can often put useful constraints on the quiescent emission (Smith et al. 2001).

Complementary programs have used SCUBA to perform sensitive searches for the quiescent emission from GRB hosts long after any afterglow emission has faded away (Barnard et al. 2003; Berger et al. 2003; Tanvir et al. 2004). These observations are generally done in good weather conditions when the source is at a high elevation.

To date, of the 21 GRB host galaxies with $850 \mu \mathrm{m}$ rms values $<1.4 \mathrm{mJy}$ (not including the new observations described here), none have had a flux density $>4 \mathrm{mJy}$. The only host galaxies significantly detected $(>3 \sigma)$ by SCUBA to date are GRBs 000210, 000418, and 010222. The tentative dearth of brighter hosts suggests that the GRBs do not in general trace the most luminous sub-millimeter galaxies. However, it should be cautioned that the current sample of bursts is small. For example, only 6 of the 21 galaxies with measured redshifts in Table 2 of Tanvir et al. (2004) have $z>2$. There also could be important selection effects since many of the bursts studied by SCUBA have had optical transients and thus may have relatively little absorption local to the source. It is also possible that the dust temperatures tend to be higher in GRB host galaxies, shifting the peak of their dust emission to shorter wavelengths and reducing the sub-millimeter flux below the current sensitivity of SCUBA (Chapman et al. 2004).

In Smith et al. (2001) we gave numerical estimates for the expected sub-millimeter emission from warm dust due to massive star formation using the formalism from Condon (1992) and Carilli \& Yun (1999). (For completeness, we can now fill in the entry for GRB 000911 in Table 2 of Smith et al. 2001, using $z=1.0585$; Price et al. 2002 to give a $\operatorname{SFR}(M \geq$ $\left.5 M_{\odot}\right)<220 M_{\odot} \mathrm{yr}^{-1}$.) However, this conversion currently has very large uncertainties due to the lack of information for key quantities such as the temperature of the dust, the spectral indices in the sub-millimeter region and the initial mass function. For example, for a given $850 \mu \mathrm{m}$ flux density, doubling the dust temperature corresponds to changing the inferred luminosity by a factor of $\sim 10$ (Blain et al. 2002). We therefore simply quote here the flux densities that we find for the new bursts. These can be directly compared to the previous results (Smith et al. 2001, 2005; Barnard et al. 2003; Berger et al. 2003; Tanvir et al. 2004).

In the future, it may be possible to combine the sub-millimeter observations with those at mid- and far-IR wavelengths, e.g. using the Spitzer Space Telescope. This 
combination - along with the known burst redshift - will allow a good modeling of the spectral energy distribution, from which the temperature and luminosity of the dust can then be determined (Blain et al. 2003). The star formation rate can then be calculated to a much higher accuracy than using the submillimeter observations alone.

\section{SCUBA observing details}

SCUBA is the sub-millimeter continuum instrument for the JCMT (for a review see Holland et al. 1999). Here we summarize only the most important features of the instrument. The observing, calibration, and reduction techniques are the same as in Smith et al. (1999, 2001).

SCUBA uses two arrays of bolometers to simultaneously observe the same region of sky, $\sim 2.3^{\prime}$ in diameter. The arrays are optimized for operations at 450 and $850 \mu \mathrm{m}$. Fully sampled maps of the $2.3^{\prime}$ region can be made by "jiggling" the array. However, for all the sources described here, we have only been looking at well-localized optical or radio transient coordinates by performing deep photometry observations using a single pixel of the arrays. The other bolometers in the arrays are used to perform a good sky noise subtraction (Archibald et al. 2002).

During an observation, the secondary is chopped between the source and sky. This is done mainly to take out small relative DC drifts between the bolometers, and also to remove any large-scale sky variations. The term "integration time" always refers to the "on+off" time, including the amount of time spent off-source. An $18 \mathrm{~s}$ integration thus amounts to a $9 \mathrm{~s}$ on-source observation time. A typical measurement consists of 50 integrations of $18 \mathrm{~s}$; we refer to this as a "run." Each observation of a source in general consists of several such runs, with focus, pointing, and calibration observations in between.

Version 1.6 of SURF$^{1}$ (Jenness \& Lightfoot 2000) was used to combine the integrations, remove anomalous spikes, flatfield the array, and subtract the signal from the sky bolometers. The zenith sky opacity was determined using the JCMT Water Vapor Monitor. This is located in the Receiver Cabin, and is pointed just slightly off the main beam. It measures the opacity every $6 \mathrm{~s}$. The results were checked using "skydips" in which the sky brightness temperature was measured at a range of elevations. The opacity was used to apply atmospheric extinction corrections to the observed target fluxes. At least one standard calibration target was observed each night to determine the absolute flux of the GRB.

A typical integration time of $2 \mathrm{~h}$ gives an rms $\sim 1.5 \mathrm{mJy}$ at $850 \mu \mathrm{m}$. However, the sensitivity depends significantly on the weather and the elevation of the source; since our ToO observations are done on short notice, sometimes these factors are less than ideal. Based on observed variations of the gain factor and signal levels we estimate typical systematic uncertainties in the absolute flux calibrations of $10 \%$ at $850 \mu \mathrm{m}$. In general the rms errors of the observations presented here are larger than this uncertainty.

\footnotetext{
${ }^{1}$ SURF is distributed by the Starlink Project.
}

\subsection{Pointing errors}

The pointing of the JCMT is checked several times during the night to ensure that it is reliable. The pointing accuracy is usually a few arcsec. However, an error in the track model used between 2000 August 25 and 2003 April 25 resulted in pointing errors that were non-negligible (shifts larger than $4^{\prime \prime}$ ) for targets with elevations above $60^{\circ}$ and over small ranges of azimuth. Since the $850 \mu \mathrm{m}$ bolometric pixel has a diffraction limited resolution of $14^{\prime \prime}$, the target remains well within the bolometric pixel. However, there will be an error in the flux that is measured. This issue can be a problem if either the source or a pointing or calibration target was affected.

For the new bursts reported here, there were small pointing problems in some of the runs for GRBs 021004 and 030226. These instances are noted in Sect. 3. None of them significantly affected the final results.

For completeness, we note that the pointing problem also affected the previously reported ToO observations of GRB 000911 (Smith et al. 2001). One of the four runs on 2000 September 17 was affected. Even if we drop that day entirely, the $850 \mu \mathrm{m}$ flux density of $0.3 \pm 1.1 \mathrm{mJy}$ measured on 2000 September 20 is still marginally inconsistent with the value of $2.31 \pm 0.91 \mathrm{mJy}$ measured by Berger et al. (2003) in their quiescent host program. (The observation on 2000 September 22 was also affected by the pointing problem, but this was not on the correct coordinates, Smith et al. 2001.)

\subsection{False positives}

The large beam size combined with the large number of distant galaxies radiating strongly at this wavelength means that in any observation there is a non-negligible chance of detecting a quiescent sub-millimeter source that is completely unrelated to the GRB.

The surface density of sub-millimeter galaxies is still somewhat uncertain (Blain et al. 2002). At $850 \mu \mathrm{m}$, the surface density of galaxies with flux densities larger than $4 \mathrm{mJy}$ is $\sim 10^{3} \mathrm{deg}^{-2}$, while the surface density of galaxies with flux densities larger than $1 \mathrm{mJy}$ is $\sim 10^{4} \mathrm{deg}^{-2}$. This gives estimates that the chance of detecting a random $\geq 4 \mathrm{mJy}$ source in any pointing is $\sim 1 \%$, while the chance of detecting a random $\geq 1 \mathrm{mJy}$ source in any pointing is $\sim 10 \%$.

The non-negligible chance of a false positive means that caution is required when using just the detection of a quiescent sub-millimeter source to claim that this must be the host galaxy to the GRB. For individual cases, confirmation of the star formation rate is needed from observations at other wavelengths. However, if it is found that many more bursts are associated with quiescent sub-millimeter sources than is expected by chance, this would be good evidence that the majority of these are true associations.

\section{Results for new SCUBA observations}

Table 1 summarizes all the $850 \mu \mathrm{m}$ SCUBA ToO observations for the new bursts. The start times are the times when the GRB was first observed on each day, and the stop times 
Table 1. SCUBA $850 \mu \mathrm{m}(350 \mathrm{GHz})$ GRB afterglow observations.

\begin{tabular}{llllllc}
\hline \hline Burst & \multicolumn{2}{c}{ SCUBA observing times } & $\begin{array}{c}\text { Time since } \\
\text { burst (days) }\end{array}$ & $\begin{array}{c}\text { Integration } \\
\text { time (s) }\end{array}$ & $\begin{array}{c}\tau_{850} \\
\text { Stop }\end{array}$ & $\begin{array}{c}\text { Afterglow } 850 \mu \mathrm{m} \\
\text { flux density (mJy) }\end{array}$ \\
\hline GRB 021004 & 20021004.622 & 20021004.665 & 0.140 & 1980 & 0.47 & $5 \pm 26$ \\
& 20021005.349 & 20021005.365 & 0.853 & 900 & 0.44 & $-4.0 \pm 5.0$ \\
& 20021006.353 & 20021006.481 & 1.913 & 4662 & 0.27 & $0.9 \pm 1.5$ \\
& 20021011.434 & 20021011.543 & 6.984 & 5400 & 0.19 & $-3.7 \pm 1.5^{a}$ \\
GRB 021211 & 20021212.488 & 20021212.520 & 1.033 & 1800 & 0.23 & $2.1 \pm 2.5$ \\
& 20021221.429 & 20021221.460 & 9.973 & 1800 & 0.40 & $-2.4 \pm 3.0$ \\
& & & & & & \\
GRB 030115 & 20030118.410 & 20030118.501 & 3.314 & 4500 & 0.34 & $0.2 \pm 1.8$ \\
& & & & & & \\
GRB 030226 & 20030226.386 & 20030226.418 & 0.245 & 1800 & 0.35 & $5.1 \pm 2.6$ \\
& 20030226.386 & 20030226.531 & 0.301 & 6498 & 0.35 & $1.2 \pm 1.3$ \\
& 20030227.412 & 20030227.477 & 1.288 & 3600 & 0.35 & $-1.9 \pm 1.8^{a}$ \\
& 20030228.489 & 20030228.564 & 2.369 & 3618 & 0.22 & $0.0 \pm 1.5^{a}$ \\
& 20030322.542 & 20030322.574 & 24.401 & 1800 & 0.48 & $-4.3 \pm 3.8$ \\
& & & & & & \\
\hline GRB 041006 & 20041006.604 & 20041006.618 & 0.098 & 720 & 0.25 & $2.0 \pm 5.0$ \\
& 20041007.374 & 20041007.590 & 0.969 & 4320 & 0.23 & $0.9 \pm 1.3$ \\
\hline
\end{tabular}

${ }^{a}$ Not corrected for small pointing error.

are when the last observation of the GRB was completed. The time since the burst uses the mid-point between these start and stop times (although it should be noted that focus, pointing, and calibration observations may have also taken place in between the start and stop times); the time is the elapsed time in the Earth frame, not the rest frame of the host galaxy. The "integration time" for each observation is the "on+off" time; only half of this is spent on-source. The zenith optical depth at $850 \mu \mathrm{m}\left(\tau_{850}\right)$ is given for the time of the observation.

The $3 \sigma$ upper limits for all the new ToO observations are shown as the filled triangles in Fig. 1.

For each burst, the $850 \mu \mathrm{m}$ data from the separate days (that have no obvious afterglow emission) was combined using a weighted mean to give the most sensitive value for the flux of the quiescent host galaxy. These results are given in Table 2 . Our observations are close enough to the time of the burst that a small amount of afterglow emission may be present in the final results. However, this contamination will be small.

In the following sections, we discuss each of these bursts in more detail.

\subsection{GRB 021004}

The long ( 100 s) GRB 021004 was detected by HETE-2 at 20021004.504 UT (Shirasaki et al. 2002). The burst was X-ray rich (Lamb et al. 2002). A fading X-ray source was found by the Chandra X-Ray Observatory whose light curve decay was variable, but whose spectrum showed no significant features (Fox et al. 2003a; Butler et al. 2003; Holland et al. 2003).
Observations starting 193 seconds after the burst detected an $R \sim 15.5$ optical afterglow (Fox et al. 2003a). The large number of lines in the optical spectrum complicated the determination of the redshift of the counterpart, but most likely it was an active starburst $R \sim 24$ galaxy at $z=2.328$ (e.g. Mirabal et al. 2003; Schaefer et al. 2003).

Unlike GRBs 990123 and 021211, where the optical light curve had a rapid initial fade before flattening to a value typical of afterglows, for GRB 021004 the afterglow had an initial decay for the first $\sim 20 \mathrm{~min}$ but then a plateau that lasted for $\sim 3 \mathrm{~h}$ (Uemura et al. 2003). There were significant variations on several time scales superposed on the later optical decay, and there were significant color and polarization changes during the afterglow (e.g. Bersier et al. 2003; Matheson et al. 2003; Rol et al. 2003; Lazzati et al. 2003). This suggests that there could have been repeated energy injections from the central source (e.g. Björnsson et al. 2004), and/or the surrounding medium may have been significantly inhomogeneous. Highly ionized lines with high relative velocities were seen in the spectrum; these may have come from shells or clumps that could have been produced by a massive stellar progenitor prior to its collapse (e.g. Mirabal et al. 2003; Schaefer et al. 2003). On larger scales, the ambient medium may have been homogeneous. A break in the optical light curve 5-9 days after the burst was most likely the jet break (Holland et al. 2003; Mirabal et al. 2003).

A variable radio and millimeter counterpart was found (Frail \& Berger 2002; Pooley 2002; Bremer \& Castro-Tirado 2002). The longer wavelength data was fitted by a simple power law $S_{v} \propto v^{\alpha}$ with $\alpha \sim 0.9$. This spectral index was considered to be unusual (Berger et al. 2002), although a similar result had 
previously been discussed for GRB 980329 (Smith et al. 1999). At $~ 1.4$ days after the burst, the peak of the multiwavelength spectrum was $\sim 3 \mathrm{mJy}$ somewhere in the range $100-1000 \mathrm{GHz}$ (Schaefer et al. 2003).

All the SCUBA $850 \mu \mathrm{m}$ observations of GRB 021004 are shown in Table 1. These expand on those given previously (Kemp et al. 2002; Wouterloot et al. 2002). On 2002 October 11 there were small pointing problems (see Sect. 2.1) with two of the SCUBA runs on the pointing calibration target PKS 0106+013. These runs do not significantly affect the results for that day.

Our first SCUBA observation of GRB 021004 started less than $3 \mathrm{~h}$ after the burst. However, the source was at a low elevation and was setting, and the weather was only fair. The upper limit was therefore much worse than normal. A second observation at a good elevation was attempted later the same day, but this had to be cut short due to fog at the summit. Again, the upper limit that was obtained was poor. Better observations were made on following days. The source was not detected by SCUBA in any of the observations.

Our non-detections are consistent with the observations at $232 \mathrm{GHz}(1.9 \pm 1.5 \mathrm{mJy}$ at 1.5 days after the burst $)$ by the IRAM Plateau de Bure Interferometer (Bremer \& CastroTirado 2002). At that time, the peak of the afterglow spectrum was only $\sim 3 \mathrm{mJy}$. Our results are also consistent with the windinteraction model of Li \& Chevalier (2003) that placed the peak of the $350 \mathrm{GHz}$ flux at $\sim 8 \mathrm{mJy}$ around $\sim 0.3$ days after the burst.

As shown in Table 2, the combination of our ToO observations puts good limits on the quiescent sub-millimeter flux of the host galaxy. These results improve slightly on those shown in Tanvir et al. (2004). The unobscured star formation rate estimated from the Ly $\alpha$ luminosity is $\sim 15 M_{\odot} /$ year (Djorgovski et al. 2002). Although this is an active starburst galaxy whose redshift is approximately at the peak for the sub-millimeter bright galaxies, our limit implies that there is not a substantial amount of obscured star formation in the host galaxy. The lack of detection of a sub-millimeter host galaxy agrees with the X-ray observations of the afterglow that show no evidence for absorption in excess of the Galactic value (Fox et al. 2003a; Butler et al. 2003; Holland et al. 2003). Some reddening due to local extinction in the host galaxy is required to explain the optical spectrum, but this is not substantial in the case of a circumstellar medium with a wind-like density profile.

\subsection{GRB 021211}

The $\sim 10$ s long FRED-like GRB 021211 was a bright, X-ray rich burst detected by HETE-2 at 20021211.471 UT (Crew et al. 2003).

Rapid optical observations detected an afterglow that faded quickly from $R \sim 14$ at $90 \mathrm{~s}$ after the burst to $R \sim 18$ after 20 min (Wozniak et al. 2002; Park et al. 2002; Fox et al. 2003b; Li et al. 2003). The initial decay rate was steep, but after $\sim 10 \mathrm{~min}$ this slowed to a value more typical of optical afterglows. The initial emission may have come from the reverse shock. Unlike GRB 021004, the optical afterglow for GRB 021211 resembled the one for GRB 990123 at
Table 2. Host galaxy fluxes for each burst determined by combining the SCUBA $850 \mu \mathrm{m}$ observations taken on separate days.

\begin{tabular}{lrc}
\hline \hline Burst & Redshift & $\begin{array}{l}\text { Host } 850 \mu \mathrm{m} \text { flux } \\
\text { density }(\mathrm{mJy})\end{array}$ \\
\hline GRB 021004 & 2.328 & $-1.4 \pm 1.0$ \\
GRB 021211 & 1.006 & $0.3 \pm 1.9$ \\
GRB 030115 & $2-2.5$ & $0.2 \pm 1.8$ \\
GRB 030226 & $\geq 1.986$ & $-0.1 \pm 0.8$ \\
GRB 041006 & 0.716 & $1.0 \pm 1.3$ \\
\hline
\end{tabular}

similar epochs, but it was 3-4 mag fainter. If it had not been for the early observations, the burst might have been classified as "optically dark."

There appear to have been some short time scale variations superposed on the overall optical light curve decay, but there was only very weak evidence for a jet break (Holland et al. 2004). A rebrightening in the optical light curve $\sim 25$ days after the burst may have been due to a supernova (Della Valle et al. 2003). An underlying $R \sim 25.2$ host galaxy was found (Della Valle et al. 2003). The redshift of the host was $z=1.006$ (Vreeswijk et al. 2003).

The source was not significantly detected in any of the radio observations that were made, putting constraints on the reverse shock (Rol \& Strom 2002; Fox et al. 2003b).

All the SCUBA $850 \mu \mathrm{m}$ observations of GRB 021211 are shown in Table 1. These expand on those given previously (Hoge et al. 2002; Fox et al. 2003b). Our first observation was made $\sim 1$ day after the burst. The source was not detected by SCUBA in either observation.

If we extrapolate from the optical spectrum using the simple synchrotron model $S_{v} \propto v^{-\beta}$ with $\beta=0.6$ as in Pandey et al. (2003), the expected $850 \mu \mathrm{m}$ flux density would have been $\sim 0.2 \mathrm{mJy}$ at 1 day after the burst. Similarly, using $\beta=0.69$ as in Holland et al. (2004) would give $\sim 0.3 \mathrm{mJy}$. Thus although GRB 021211 had a detectable optical reverse shock, it is not surprising that it was not detected in the sub-millimeter given the faintness of the optical afterglow at the times the SCUBA observations were made, and the lack of radio detection at any time.

As shown in Table 2, the combination of our ToO observations limits the quiescent sub-millimeter flux of the host galaxy. Given the relatively low redshift, and the lack of extinction in the host galaxy (Holland et al. 2004), it is not surprising that it is not a bright sub-millimeter source. The fact that no SCUBA source was detected confirms that this burst had an intrinsically faint afterglow, rather than one that was heavily absorbed by dust in the host galaxy.

\subsection{GRB 030115}

The $\sim 20$ s long GRB 030115 was detected by HETE-2 at 20030115.141 UT (Kawai et al. 2003). Optical observations simultaneous with the burst did not detect the source, with an upper limit of $R \sim 10$ (Castro-Tirado et al. 2003). Early optical afterglow searches did not detect a counterpart, but a fading 
infrared source was found (Levan et al. 2003; Vrba et al. 2003; Masetti et al. 2003; Kato et al. 2003). A possible $R=25.2$ host galaxy was found (Garnavich 2003; Dullighan et al. 2004). Although no spectroscopic redshift has been reported to date, the photometric redshift lies in the range $z=2-2.5$ (Levan et al. 2005). A faint $(<0.1 \mathrm{mJy})$ radio source was detected at the afterglow location (Frail \& Berger 2003; Rol \& Wijers 2003).

Since the afterglow was faint, and there were limited observations being made at other wavelengths, we only performed one SCUBA observation of GRB 030115. As shown in Table 1 - which updates the result of Hoge et al. (2003) - the source was not detected at $850 \mu \mathrm{m}$. This is consistent with an observation made by MAMBO on IRAM a few hours earlier that found $2.9 \pm 1.6 \mathrm{mJy}$ at $1.2 \mathrm{~mm}$ (Bertoldi et al. 2003).

While the red $R-K$ color of the afterglow and host galaxy indicates significant reddening (Levan et al. 2005), the nondetection by SCUBA, albeit with a fairly large uncertainty, suggests that it is not a massive star-bursting, dusty galaxy.

\subsection{GRB 030226}

The long (>100 s) GRB 030226 was detected by HETE-2 at 20030226.157 UT (Suzuki et al. 2003). Optical observations simultaneous with the burst did not detect the source, placing an upper limit of $R=11.5$ on the reverse shock emission (Klose et al. 2004). A fading optical counterpart was found $2.6 \mathrm{~h}$ after the burst (Fox et al. 2003c; Price et al. 2003a), and a fading $\mathrm{X}$-ray source was found by the Chandra X-Ray Observatory (Pedersen et al. 2003; Klose et al. 2004). The optical afterglow started faint and faded quickly, with an achromatic break $\sim 0.8$ days after the burst indicating a jetted explosion (Klose et al. 2004). The redshift of the optical counterpart was determined to be $\geq 1.986$ (Ando et al. 2003; Price et al. 2003b; Chornock \& Filippenko 2003; Klose et al. 2004). No optical host galaxy was detected down to a limit of $R=26$ (Klose et al. 2004).

As shown in Table 1, our first observation of GRB 030226 with SCUBA started $5.5 \mathrm{~h}$ after the burst. We give two results for the observations on the first night. The first 100 integrations indicated the presence of a source at the $\sim 2 \sigma$ level. However, the complete data set for that night did not confirm a significant detection, possibly because the sub-millimeter flux was already falling. The observations on following nights did not detect the source.

There was a small pointing problem (see Sect. 2.1) with one of the four SCUBA runs on 2003 February 27. On 2003 February 28, there was a problem with a pointing calibration observation of $1156+295$. These runs do not significantly affect the results for those days. There were no problems on 2003 February 26 that could explain the possibly varying source.

Millimeter observations with the Plateau de Bure Interferometer did not detect the source (Pandey et al. 2004), but these did not start until 1.8 days after the burst. From the radio catalog of Frail et al. (2003b), VLA observations 0.12 days after the burst did not detect the source, with a flux density of $-0.058 \pm 0.074 \mathrm{mJy}$ at $8.46 \mathrm{GHz}$. A $\sim 0.1 \mathrm{mJy}$ source was detected at $8.46 \mathrm{GHz}$ starting at 1.09 days after the burst. Ryle observations 0.94 days after the burst gave $0.19 \pm 0.22 \mathrm{mJy}$ at $15 \mathrm{GHz}$, and there was no detection with OVRO 2.27 days after the burst at $98 \mathrm{GHz}$. Given the sparse longer wavelength coverage and larger rms of the first VLA observation, there is limited information regarding the reality of the possible SCUBA source at early times.

If we extrapolate from the optical spectrum using the simple synchrotron model $S_{v} \propto v^{-\beta}$ with $\beta=0.55$ as in Fig. 2 of Pandey et al. (2004), the expected $850 \mu \mathrm{m}$ flux density would have been $\sim 4$ mJy at 0.62 days after the burst. A similar flux is expected if we instead extrapolate using $\beta=0.7$ at the time of the optical light curve break as in Fig. 8 of (Klose et al. 2004). Thus our observations - that were made earlier than these times when the optical afterglow was brighter - appear to lie a little below these extrapolations.

Klose et al. (2004) noted that the optical/infrared light curves had some deviations from the best fit model. For example, the $K$-band flux was 0.4 mag above the best fit model at 0.18 days after the burst. Although the fluctuations had a lower amplitude than in other bursts, they might indicate expanding shells around a massive Wolf-Rayet star. The optical/infrared light curves were very sparsely sampled at early times, but suggest that deviations were not present at the time of our first SCUBA observation. Thus it is not clear if our initial SCUBA observations can be explained as being due to a short-lived fluctuation. If we assume that there was an early sub-millimeter source that faded quickly, this would favor the scenario where the fireball was interacting with a stellar wind rather than a homogeneous medium. This would be consistent with the suggestion of a Wolf-Rayet progenitor.

As shown in Table 2, the combination of our ToO observations puts good limits on the quiescent $850 \mu \mathrm{m}$ flux of the host galaxy. Similarly, at $450 \mu \mathrm{m}$ the combined flux density is $1 \pm 10 \mathrm{mJy}$. Although the redshift of the galaxy is at approximately the peak for sub-millimeter bright galaxies, the host for GRB 030226 is not a bright dusty galaxy. This agrees with the broadband optical spectrum, which shows no evidence for additional reddening by dust in the host galaxy, and the low polarization of the afterglow, which argues against substantial dust extinction (Klose et al. 2004).

\subsection{GRB 041006}

The $\sim 25$ s long GRB 041006 was detected by HETE-2 at 20041006.513 UT (Galassi et al. 2004). The burst was reminiscent of GRB 030329 in shape and spectral properties, but was 20 times fainter. A fading X-ray source was detected by Chandra (Butler et al. 2004). A fading $\sim 17$ th mag optical counterpart was found starting a few minutes after the burst (e.g. da Costa et al. 2004; Price et al. 2004a, 2004b; Maeno et al. 2004). There was a break in the afterglow light curve $\sim 7 \mathrm{~h}$ after the burst (Kahharov et al. 2004; D'Avanzo et al. 2004). The break was consistent with being achromatic, suggesting the explosion was jetted. The redshift of the optical counterpart was determined to be 0.716 (Fugazza et al. 2004; Price et al. 2004c). A host galaxy with $R \gtrsim 25$ was found (Fynbo et al. 2004; 
Covino et al. 2004). Radio observations with the VLA starting at 0.74 days after the burst did not detect a source at $4.86 \mathrm{GHz}$ or $8.46 \mathrm{GHz}$, with rms values of $0.059 \mathrm{mJy}$ and $0.041 \mathrm{mJy}$ respectively (Soderberg \& Frail 2004).

As shown in Table 1, our first observation of GRB 041006 with SCUBA started $2.2 \mathrm{~h}$ after the burst. The source was not detected (Barnard et al. 2004a). Unfortunately, the source was setting, so it was not possible to perform a longer observation to get a lower rms or to look for any evolution in the source flux. Longer observations the following night also did not detect the source (Barnard et al. 2004b).

The $850 \mu \mathrm{m}$ flux density for GRB 030329 was $\sim 31 \mathrm{mJy}$ during the first week. Therefore, our observations of GRB 041006 are consistent with this source being similar to GRB 030329, but with a flux that is 20 times lower.

As shown in Table 2, the combination of our ToO observations gives a good limit to the quiescent sub-millimeter flux of the host galaxy. Given the relatively low redshift, it is not surprising that it is not a bright sub-millimeter source.

\section{Discussion}

The observations of GRBs 021004, 021211, 030226, and 041006 all started within $\sim 1$ day of the burst, but did not detect any significant sub-millimeter emission from the reverse shock and/or afterglow. These observations put some constraints on the models for the early emission, although the generally poor observing conditions and/or the faintness of these afterglows at other wavelengths limit the inferences that can be drawn from these lack of detections. However, Fig. 1 shows that we have begun to meaningfully sample the sub-millimeter emission at early times where there can be significant differences in the evolution of the sub-millimeter flux in the wind and ISM scenarios.

None of the new GRBs studied here had a significant submillimeter emission from the host galaxy. In particular, the host galaxies for GRBs 030226 and 041006 had $850 \mu \mathrm{m} \mathrm{rms}$ values $<1.4 \mathrm{mJy}$. These can be added to the compilation in Table 2 of Tanvir et al. (2004) to give 23 host galaxies with rms values $<1.4 \mathrm{mJy}$. The redshifts of GRBs 030115 and 030226 were $\gtrsim 2$. Adding all the bursts presented here and GRB 030329 (Smith et al. 2005) to Table 2 of Tanvir et al. (2004), there are now $8 / 26$ host galaxies with measured redshifts $\gtrsim 2$. The lack of new detections adds to the indication that GRBs are not closely linked to the most luminous dusty star-forming galaxies. However, as was pointed out in Sect. 1.2, the sample size remains small. There is also the possible selection effect that this may not be a representative sample of the whole host galaxy population because many of the GRBs studied by SCUBA to date have had optical transients. It is also possible that GRB host galaxies tend to have hotter dust, shifting the peak of their far-IR emission to shorter wavelengths.

Observations of new bursts are continuing to produce surprises, and there is much left to learn about GRB afterglows and host galaxies. To obtain a complete picture of their nature will require the careful study of many bursts to expand our sample. Sub-millimeter observations with a $\sim$ mJy sensitivity are a key component to the multi-wavelength coverage. To this end, our program of ToO observations using SCUBA is ongoing.

\section{The future with Swift}

Only $\sim 3$ bursts per year have been observed by the SCUBA ToO program over the past 7 years. This has been due to a combination of (1) the lack of well-localized sources in regions of the sky accessible to SCUBA; (2) downtimes to SCUBA; and (3) poor weather over this period.

This situation should improve significantly over the coming years because of the rapid burst location capabilities of Swift (Gehrels et al. 2004). Swift should localize $\sim 100$ bursts per year (its gamma-ray instrument will be the most sensitive GRB detector flown to date, and so the number of bursts it will detect depends on the uncertain number of faint sources). This will allow us to focus our SCUBA observations on the bursts that appear to be the most interesting and those that occur in good observing conditions.

Using its gamma-ray imaging capabilities, Swift will distribute $\sim 4^{\prime}$ localizations within $8 \mathrm{~s}$. The satellite will then automatically repoint so that the source is in the field of view of the on-board X-ray and UV-optical instruments. If an X-ray counterpart is present, coordinates with an accuracy of $\sim 5^{\prime \prime}$ will be available $\sim 96 \mathrm{~s}$ after the burst. If an optical counterpart is present, coordinates with an accuracy of $\sim 0.3^{\prime \prime}$ will be available $\sim 243 \mathrm{~s}$ after the burst. Even if only an X-ray source is present, we will be able to immediately use SCUBA in the photometry mode (the $850 \mu \mathrm{m}$ bolometric pixel has a diffraction limited resolution of $\left.14^{\prime \prime}\right)$. Thus we will be able to look for sources that may be hard to detect otherwise if the redshift is large and/or if the optical extinction is large.

It is also exciting that Swift will be able to localize bursts that last less than $1 \mathrm{~s}$ : these may have different progenitors and counterpart behaviors from the objects studied to date (Kouveliotou et al. 1993; Panaitescu et al. 2001). All of the bursts studied by SCUBA to date have been in the long duration class.

The results presented here demonstrate that we can perform observations with SCUBA shortly after a burst is reported. The possible $(\sim 2 \sigma)$ source in the early observation of GRB 030226 provides encouragement for future similar observations to study the reverse shock and early fluctuations in the afterglow light curve. We are therefore in an excellent position to take advantage of Swift.

Acknowledgements. The James Clerk Maxwell Telescope is operated by The Joint Astronomy Centre on behalf of the Particle Physics and Astronomy Research Council of the UK, The Netherlands Organisation for Scientific Research, and the National Research Council of Canada.

We thank the JCMT Directors Ian Robson and Gary Davis for authorizing the ToO observations. We are indebted to all the observers whose time was displaced by these observations, and acknowledge the dedicated efforts of the JCMT telescope operators for their valuable assistance with the observations. We thank the referee for a prompt review. 
We are grateful to Scott Barthelmy and Paul Butterworth for maintaining the GRB Coordinates Network (GCN), and to the other groundbased observers for the rapid dissemination of their burst results. We acknowledge the data analysis facilities provided by the Starlink Project which is run by CCLRC on behalf of PPARC.

Some of the radio data referred to in this paper were drawn from the GRB Large Program at the VLA, http://www.vla.nrao.edu/ astro/prop/largeprop/. The National Radio Astronomy Observatory is a facility of the National Science Foundation operated under cooperative agreement by Associated Universities, Inc. The work at Rice University was supported in part by AFOSR/NSF grant number NSF AST-0123487.

\section{References}

Ando, M., Ohta, K., Watanabe, C., et al. 2003, GCN, 1884

Archibald, E. N., Jenness, T., Holland, W. S., et al. 2002, MNRAS, 336,1

Barnard, V. E., Blain, A. W., Tanvir, N. R., et al. 2003, MNRAS, 338,1

Barnard, V., Schieven, G., Tilanus, R., et al. 2004a, GCN, 2774

Barnard, V., Schieven, G., Tilanus, R., et al. 2004b, GCN, 2786

Berger, E., Sari, R., \& Frail, D. A. 2000, ApJ, 545, 56

Berger, E., Kulkarni, S. R., \& Frail, D. A. 2002, GCN, 1612

Berger, E., Cowie, L. L., Kulkarni, S. R., et al. 2003, ApJ, 588, 99

Bersier, D., Stanek, K. Z., Winn, J. N., et al. 2003, ApJ, 584, L43

Bertoldi, F., Frail, D. A., Berger, E., et al. 2003, GCN, 1835

Björnsson, G., Gudmundsson, E. H., \& Jóhannesson, G. 2004, ApJ, 615, L77

Blain, A. W., \& Natarajan, P. 2000, MNRAS, 312, L35

Blain, A. W., Smail, I., Ivison, R. J., \& Kneib, J.-P. 1999, MNRAS, 302,632

Blain, A. W., Smail, I., Ivison, R. J., Kneib, J.-P., \& Frayer, D. T. 2002, Phys. Rep., 369, 111

Blain, A. W., Barnard, V. E., \& Chapman, S. C. 2003, MNRAS, 338, 733

Bloom, J. S., Frail, D. A., Kulkarni, S. R., et al. 1998, ApJ, 508, L21

Bremer, M., \& Castro-Tirado, A. J. 2002, GCN, 1590

Butler, N. R., Marshall, H. L., Ricker, G. R., et al. 2003, ApJ, 597, 1010

Butler, N., Vanderspek, R., Marshall, H. L., et al. 2004, GCN, 2808

Carilli, C. L., \& Yun, M. S. 1999, ApJ, 513, L13

Castro-Tirado, A. J., Mateo Sanguino, T. J., de Ugarte Postigo, A., et al. 2003, GCN, 1826

Chapman, S. C., Blain, A. W., Ivison, R. J., \& Smail, I. R. 2003, Nature, 422, 695

Chapman, S. C., Smail, I., Blain, A. W., \& Ivison, R. J. 2004, ApJ, 614,671

Chapman, S. C., Blain, A. W., Smail, I., \& Ivison, R. J. 2005, ApJ, 622,772

Chevalier, R. A., \& Li, Z.-Y. 2000, ApJ, 536, 195

Chornock, R., \& Filippenko, A. V. 2003, GCN, 1897

Christensen, L., Hjorth, J., \& Gorosabel, J. 2004, A\&A, 425, 913

Condon, J. J. 1992, ARA\&A, 30, 575

Conselice, C. J., Chapman, S. C., \& Windhorst, R. A. 2003, ApJ, 596, L5

Covino, S., Malesani, D., Tagliaferri, G., et al. 2004, GCN, 2803

Crew, G. B., Lamb, D. Q., Ricker, G. R., et al. 2003, ApJ, 599, 387

da Costa, G., Noel, N., \& Price, P. A. 2004, GCN, 2765

D'Avanzo, P., Covino, S., Antonelli, L. A., et al. 2004, GCN, 2788

Della Valle, M., Malesani, D., Benetti, S., et al. 2003, A\&A, 406, L33
Djorgovski, S. G., Barth, A., Price, P., et al. 2002, GCN, 1620

Dullighan, A., Ricker, G., Butler, N., \& Vanderspek, R. 2004, in Gamma-Ray Bursts: 30 Years of Discovery: Gamma-Ray Burst Symposium, ed. E. E. Fenimore, \& M. Galassi, AIP Conf. Proc., 727,467

Dunlop, J. S., McLure, R. J., Yamada, T., et al. 2004, MNRAS, 350, 769

Fox, D. W., Yost, S., Kulkarni, S. R., et al. 2003a, Nature, 422, 284

Fox, D. W., Price, P. A., Soderberg, A. M., et al. 2003b, ApJ, 586, L5

Fox, D. W., Chen, H. W., \& Price, P. A. 2003c, GCN, 1879

Frail, D. A., \& Berger, E. 2002, GCN, 1574

Frail, D. A., \& Berger, E. 2003, GCN, 1827

Frail, D. A., Berger, E., Galama, T., et al. 2000, ApJ, 538, L129

Frail, D. A., Bertoldi, F., Moriarty-Schieven, G. H., et al. 2002, ApJ, 565,829

Frail, D. A., Yost, S. A., Berger, E., et al. 2003a, ApJ, 590, 992

Frail, D. A., Kulkarni, S. R., Berger, E., \& Wieringa, M. H. 2003b, ApJ, 125, 2299

Fugazza, D., Fiore, F., Covino, S., et al. 2004, GCN, 2782

Fynbo, J. P. U., Jakobsson, P., Møller, P., et al. 2003, A\&A, 406, L63

Fynbo, J. P. U., Jensen, B. L., Pedersen, K., et al. 2004, GCN, 2802

Galama, T. J., Wijers, R. A. M. J., Bremer, M., et al. 1998, ApJ, 500, L97

Galama, T. J., Briggs, M. S., Wijers, R. A. M. J., et al. 1999, Nature, 398, 394

Galassi, M., Ricker, G., Atteia, J.-L., et al. 2004, GCN, 2770

Garnavich, P. 2003, GCN, 1848

Gehrels, N., Chincarini, G., Giommi, P., et al. 2004, ApJ, 611, 1005

Granot, J., \& Sari, R. 2002, ApJ, 568, 820

Granot, J., Piran, T., \& Sari, R. 2000, ApJ, 534, L163

Granot, J., Nakar, E., \& Piran, T. 2003, Nature, 426, 138

Harrison, F. A., Yost, S. A., Sari, R., et al. 2001, ApJ, 559, 123

Hoge, J., Willott, C., Grimes, J., Tilanus, R., \& Moriarty-Schieven, G. 2002, GCN, 1742

Hoge, J. C., Stevens, J. A., Moriarty-Schieven, G., \& Tilanus, R. P. J. 2003, GCN, 1832

Holland, S. T., Weidinger, M., Fynbo, J. P. U., et al. 2003, AJ, 125, 2291

Holland, S. T., Bersier, D., Bloom, J. S., et al. 2004, AJ, 128, 1955

Holland, W. S., Robson, E. I., Gear, W. K., et al. 1999, MNRAS, 303, 659

Inoue, S., Omukai, K., \& Ciardi, B. 2005, MNRAS, submitted [arXiv: astro-ph/0502218]

Jenness, T., \& Lightfoot, J. F. 2000, Starlink User Note 216, Starlink Project, CLRC

Kahharov, B., Asfandiyarov, I., Ibrahimov, M., et al. 2004, GCN, 2775

Kato, D., Nagata, T., \& Kawai, N. 2003, GCN, 1830

Kawai, N., Ricker, G., Atteia, J.-L., et al. 2003, GCN, 1816

Kemp, J., Fiege, J., Coppin, K., et al. 2002, GCN, 1619

Klose, S., Greiner, J., Rau, A., et al. 2004, AJ, 128, 1942

Kouveliotou, C., Meegan, C. A., Fishman, G. J., et al. 1993, ApJ, 413, L101

Kulkarni, S. R., Frail, D. A., Sari, R., et al. 1999, ApJ, 522, L97

Lamb, D., Ricker, G., Atteia, J.-L., et al. 2002, GCN, 1600

Lazzati, D., Covino, S., di Serego Alighieri, S., et al. 2003, A\&A, 410, 823

Levan, A., Merrill, M., Rol, E., et al. 2003, GCN, 1818

Levan, A., et al. 2005, in press

Li, W., Filippenko, A. V., Chornock, R., \& Jha, S. 2003, ApJ, 586, L9

Li, Z.-Y., \& Chevalier, R. A. 2003, ApJ, 589, L69

Livio, M., \& Waxman, E. 2000, ApJ, 538, 187

Maeno, S., Sonoda, E., Matsuo, Y., \& Yamauchi, M. 2004, GCN, 2772

Masetti, N., Bartolini, C., Bernabei, S., et al. 2000, A\&A, 359, L23 
Masetti, N., Palazzi, E., Pian, E., et al. 2003, GCN, 1823

Matheson, T., Garnavich, P. M., Foltz, C., et al. 2003, ApJ, 582, L5

Mészáros, P. 2002, ARA\&A, 40, 137

Mirabal, N., Halpern, J. P., Chornock, R., et al. 2003, ApJ, 595, 935

Panaitescu, A., \& Kumar, P. 2000, ApJ, 543, 66

Panaitescu, A., \& Kumar, P. 2004, MNRAS, 350, 213

Panaitescu, A., Kumar, P., \& Narayan, R. 2001, ApJ, 561, L171

Pandey, S. B., Anupama, G. C., Sagar, R., et al. 2003, A\&A, 408, L21

Pandey, S. B., Sagar, R., Anupama, G. C., et al. 2004, A\&A, 417, 919

Park, H. S., Williams, G., \& Barthelmy, S. 2002, GCN, 1736

Pedersen, K., Fynbo, J., Hjorth, J., et al. 2003, GCN, 1924

Piran, T. 1999, Phys. Rep., 314, 575

Pooley, G. 2002, GCN, 1604

Price, P. A., Berger, E., Kulkarni, S. R., et al. 2002, ApJ, 573, 85

Price, P. A., Fox, D. W., \& Chen, H. W. 2003a, GCN, 1880

Price, P. A., Fox, D. W., \& Djorgovski, S. G., et al. 2003b, GCN, 1889

Price, P. A., da Costa, G., \& Noel, N. 2004a, GCN, 2766

Price, P. A., da Costa, G., \& Noel, N. 2004b, GCN, 2771

Price, P. A., Roth, K., Rich, J., et al. 2004c, GCN, 2791

Ramirez-Ruiz, E., Trentham, N., \& Blain, A. W. 2002, MNRAS, 329, 465

Rol, E., \& Strom, R. 2002, GCN, 1777

Rol, E., \& Wijers, R. 2003, GCN, 1867

Rol, E., Wijers, R. A. M. J., Fynbo, J. P. U., et al. 2003, A\&A, 405, L23

Sari, R., \& Mészáros, P. 2000, ApJ, 535, L33

Sari, R., Piran, T., \& Narayan, R. 1998, ApJ, 497, L17

Schaefer, B. E., Gerardy, C. L., Höflich, P., et al. 2003, ApJ, 588, 387

Shirasaki, Y., Graziani, C., Matsuoka, M., et al. 2002, GCN, 1565
Smith, I. A., Tilanus, R. P. J., Van Paradijs, J., et al. 1999, A\&A, 347, 92

Smith, I. A., Van Paradijs, J., Tilanus, R. P. J., et al. 2000, in GammaRay Bursts: 5th Huntsville Symposium, ed. R. M. Kippen, R. S. Mallozzi, \& G. J. Fishman (New York: AIP), 326

Smith, I. A., Tilanus, R. P. J., Wijers, R. A. M. J., et al. 2001, A\&A, 380,81

Smith, I. A., Tilanus, R. P. J., Tanvir, N., et al. 2005, A\&A, submitted [arXiv: astro-ph/0503573]

Soderberg, A. M., \& Frail, D. A. 2004, GCN, 2787

Suzuki, M., Shirasaki, Y., Graziani, C., et al. 2003, GCN, 1888

Tanvir, N. R., Barnard, V. E., Blain, A. W., et al. 2004, MNRAS, 352, 1073

Trentham, N., Ramirez-Ruiz, E., \& Blain, A. W. 2002, MNRAS, 334, 983

Uemura, M., Kato, T., Ishioka, R., \& Yamaoka, H. 2003, PASJ, 55, L31

Van Paradijs, J., Kouveliotou, C., \& Wijers, R. A. M. J. 2000, ARA\&A, 38, 379

Vrba, F., Luginbuhl, C., \& Henden, A. 2003, GCN, 1822

Vreeswijk, P., Fruchter, A., Hjorth, J., \& Kouveliotou, C. 2002, GCN, 1785

Wijers, R. A. M. J., \& Galama, T. J. 1999, ApJ, 523, 177

Wouterloot, J., Davis, G., Naylor, D., Tilanus, R., \& Moriarty-Schieven, G. 2002, GCN, 1627

Wozniak, P., Vestrand, W. T., Starr, D., et al. 2002, GCN, 1757

Yost, S. A., Frail, D. A., Harrison, F. A., et al. 2002, ApJ, 577, 155

Yost, S. A., Harrison, F. A., Sari, R., \& Frail, D. A. 2003, ApJ, 597, 459 